non-invasively without prior tumor mutational knowledge. The overall utDNA level for each patient was represented by the nonsilent mutation with the highest variant allele fraction after removing germline variants. Urine was similarly analyzed from 15 healthy adults. Tumor mutational burden (TMB) was inferred from the number of non-silent mutations detected in urine cell-free DNA by applying a linear relationship derived from TCGA whole exome sequencing of 409 MIBC tumors. RESULTS/ANTICIPATED RESULTS: utDNA levels were significantly higher in patients with residual disease detected in their surgical pathology compared to those who achieved a pathologic complete response $(\mathrm{P}=0.002)$. Using an optimal utDNA threshold to define MRD detection, positive utDNA MRD significantly predicted the absence of pathologic complete response with a sensitivity of $81 \%$ and specificity of $81 \%$. Positive utDNA MRD also portended significantly worse progression-free survival $(\mathrm{HR}=7.4 ; \mathrm{P}=0.03)$ compared to negative utDNA MRD. Furthermore, we applied a linear relationship (Pearson $\mathrm{r}=0.84 ; \mathrm{P}<0.0001$ ) to identify patients with high inferred TMB who may have been candidates for early immune checkpoint blockade. DISCUSSION/SIGNIFICANCE OF FINDINGS: utDNA MRD analysis prior to surgery correlated significantly with pathologic response and progression-free survival, which may help select patients for bladder-sparing treatment. utDNA can also non-invasively infer TMB, which could facilitate personalized adjuvant therapy for patients in the future.

00006

Yield of routine PET/CT surveillance imaging after primary surgical treatment for asymptomatic patients with high-risk stage II/III melanoma

Zachary J. Jaeger, Rachel E. Reed, Abigail R. Barker, Joyce C. Mhlanga, Lynn A. Cornelius and Ryan C. Fields

All at Washington University School of Medicine

ABSTRACT IMPACT: The results of this research may influence NCCN guidelines on PET/CT surveillance for this population of patients for whom the guidelines are currently vague. OBJECTIVES/GOALS: We aim to quantify and describe the yield of surveillance PET/CT for detecting asymptomatic recurrence of melanoma after primary surgical treatment for stages IIB, IIC, and IIIA. Our goal is to provide evidence to inform appropriate guidelines for scheduling surveillance PET/CT for this population. METHODS/STUDY POPULATION: This is a retrospective study of patients who have undergone a PET/CT at Barnes-Jewish Hospital. Data will be collected in our Research Electronic Data Capture (REDCap) database. The sample size is 158. Data analysis will be explanatory for the yield of imaging and description of false positives and additional unnecessary workup. Survival endpoints will be reported and multivariate analysis with subgroups will be performed for predictors of PET/CT results. Cost-efficiency analysis will be conducted in collaboration with the Center for Health Economics and Policy (CHEP), with emphasis on a patient-oriented perspective. RESULTS/ANTICIPATED RESULTS: To date, we have collected data for 158 patients, with approximately equal numbers of each stage (56 IIB, 54 IIC, 48 IIIA). Due to lack of clear guidelines for this population, there is significant variation of imaging schedules and results between similar patients or groups of patients. This makes it difficult to anticipate results. Based on clinical experience, literature review, and preliminary data, we may anticipate lower yield of routine PET/CT for the detection of asymptomatic recurrence, with frequent false negatives and occasional false positives, in addition to detection and further workup of benign processes. DISCUSSION/SIGNIFICANCE OF FINDINGS: The high-risk stages IIB, IIC, and IIIA currently occupy a gray area where surveillance PET/CT remains controversial. For this group of patients, it is important to weigh the benefits of early detection and risks of false positives, unnecessary workup and anxiety, and cost.

\section{Basic Science}

24780

Investigating the role of mycobacterial lipid antigens and CD1-restricted $\mathrm{T}$ cells in host-protective tuberculosis immunity using a guinea pig model*

Macallister Harris ${ }^{1}$, James Dilisio ${ }^{2}$, Hadley Gary ${ }^{1}$, Edward Chan ${ }^{3}$, D. Branch Moody ${ }^{4}$ and Brendan Podell ${ }^{1}$

${ }^{1}$ Colorado State University; ${ }^{2}$ University of Colorado of Denver;

${ }^{3}$ University of Colorado Anschutz Medical Campus; ${ }^{4}$ Harvard

Medical School

ABSTRACT IMPACT: Examining lipid immunity for Mycobacterium tuberculosis in a translatable Guinea pig model may serve as a critical foundation for the creation of an efficacious human lipid based vaccine against tuberculosis. OBJECTIVES/ GOALS: CD1 is a group of glycoproteins on antigen-presenting cells (APCs) that present lipid antigens to T cells. Mycobacterium tuberculosis (Mtb) has a lipid-rich cell wall which is essential for the pathogenesis of tuberculosis. Our goal is to determine the frequency, phenotypes, and functionality of CD1 T cells against Mtb using the guinea pig model. METHODS/STUDY POPULATION: Guinea pigs serve as the best translational model for CD1 immunology as they have both group 1 and group $2 \mathrm{CD} 1$ complexes, comparable to human CD1. We performed ex-vivo and in-vivo experiments to analyze lipid antigen-specific CD1 T cell responses with Mtb infection. Assays to detect lipid-specific CD1 T cell activation include cellular proliferation, cytotoxicity assays, and interferon-gamma (IFN $\gamma$ ) release assay (Elispot) using both synthetic and Mtb-derived lipids. We isolated and characterized CD1 T cells using tetramerized CD1 complexes loaded with specific Mtb lipids. Spatial interaction between lipid loaded CD1 APCs with CD1 T cells were demonstrated by immunohistochemistry (IHC). Lastly, we will investigate the impact of lipid-based immunology via knockdown and overexpression of CD1 complexes. RESULTS/ANTICIPATED RESULTS: The cytotoxicity assay demonstrated that the CD1b1 and CD1b3 complexes play roles in the presentation of Mtb lipids, specifically glucose monomycolate, and mycolic acid, as noted by $\mathrm{T}$ cell killing of fibroblasts that express specific CD1 complexes that can present Mtb lipids. Similarly, cellular proliferation exhibited lipid specific $\mathrm{T}$ cell proliferation. IFN $\gamma$ production by the stimulated CD1-restricted $\mathrm{T}$ cells (Elispot) was weak indicating CD1 $\mathrm{T}$ cells may not produce IFN $\gamma$. IHC successfully showed CD1 APCs in lungs and spleens of infected guinea pigs. It is anticipated that knocking out CD1 expression will lead to impaired immunity, and increase severity of disease as noted by pathologic lesions/bacterial burden, and systemic spread; in contrast, $\mathrm{CD} 1$ enhancement will limit the severity of tuberculosis. DISCUSSION/SIGNIFICANCE OF FINDINGS: We characterized 